\title{
The Effectiveness of Exercise Therapy on Scapular Position and Motion in Individuals With Scapular Dyskinesis: Systematic Review Protocol
}

\author{
Afsun Nodehi Moghadam¹, BS, MSc, PhD; Kianoush Abdi², BS, MSc, PhD; Mohsen Shati ${ }^{3}$, MD, PhD; Shohreh \\ Noorizadeh Dehkordi ${ }^{4}$, BS, MSc, PhD; Abbas Ali Keshtkar ${ }^{5}$, MD, PhD; Zahra Mosallanezhad ${ }^{1}$, BS, MSc, PhD \\ ${ }^{1}$ Department of Physiotherapy, University of Social Welfare and Rehabilitation Sciences, Tehran, Islamic Republic Of Iran \\ ${ }^{2}$ Department of Rehabilitation Management, University of Social Welfare and Rehabilitation Sciences, Tehran, Islamic Republic Of Iran \\ ${ }^{3}$ Department of Aging, University of Social Welfare and Rehabilitation Sciences, Tehran, Islamic Republic Of Iran \\ ${ }^{4}$ Department of Physiotherapy, School of Rehabilitation Sciences, Iran University of Medical Sciences, Tehran, Islamic Republic Of Iran \\ ${ }^{5}$ Department of Health Sciences Education Development, School of Public Health, Tehran University of Medical Sciences, Tehran, Islamic Republic \\ Of Iran
}

\section{Corresponding Author:}

Afsun Nodehi Moghadam, BS, MSc, PhD

Department of Physiotherapy

University of Social Welfare and Rehabilitation Sciences

Koodakyar Ave

Evin

Tehran,

Islamic Republic Of Iran

Phone: 982122180084 ext 21

Email: afsoonnodehi@gmail.com

\begin{abstract}
Background: Scapular dyskinesis is an alteration in normal scapular position and motion. Some researchers believe that altered kinematics of the scapula subsequent to dysfunction or weakness of scapular stabilizing muscles contributes to impingement syndrome. Scapular muscle exercises are included in the rehabilitation of patients with subacromial impingement syndrome and scapular dyskinesis because the muscular system is one of the major contributors of scapular positioning both at rest and during shoulder movement, but there is considerable uncertainty relating to the relative effectiveness of such approaches on changing scapular position and motion.
\end{abstract}

Objective: The aim of this systematic review protocol is to evaluate the effectiveness of exercise therapy on scapular position and motion in individuals with scapular dyskinesis.

Methods: A systematic review will be conducted using PubMed, Scopus, Web of Science, Elsevier, Ovid, ProQuest, Physiotherapy Evidence Database, and Cochrane Library. The reference lists of articles, other reviews, gray literature, and key journals will be searched for relevant articles. Clinical trials reporting the effect of therapeutic exercises (scapular strengthening exercise, scapular stabilization exercise, scapular muscle stretching) with the aims of changing scapular position and motion in individuals with scapular dyskinesis will be included. Two independent reviewers will select studies, extract data, and assess the quality of primary studies. Any disagreement during the selection of studies will be discussed and decided by the whole team.

Results: This systematic review began in December 2016 and is currently in progress. The findings will be synthesized to determine the effectiveness of recommended therapeutic exercise on scapular position and motion in individuals with scapular dyskinesis.

Conclusions: This is the first systematic review protocol aiming to assess the effectiveness of exercise therapy in individuals with scapular dyskinesis. The systematic review doesn't require ethics approval because all data used will be provided from published documents. The results of this study will be published in a peer-reviewed journal.

Trial Registration: PROSPERO CRD42017053923; https://www.crd.york.ac.uk/prospero/display_record.php?RecordID=53923 (Archived by WebCite at http://www.webcitation.org/6uzq32T02) 
(JMIR Res Protoc 2017;6(12):e240) doi: 10.2196/resprot.8011

\section{KEYWORDS}

scapula; exercise therapy; rehabilitation; systematic review; protocol

\section{Introduction}

\section{Background}

Elevation of the arm requires normal function of the rotator cuff to stabilize the humeral head in the glenoid fossa and coordinated motion of the scapula [1]. During arm elevation, the scapula upwardly rotates, posteriorly tilts, and externally rotates [2,3]. These scapular motions depend on normal function of the scapular stabilizers including trapezius, rhomboid, and serratus anterior muscles [1].

Scapular dyskinesis is an alteration in normal scapular position and motion. It is characterized by prominence of the scapular medial border and/or inferior angle relative to the thoracic cage in the static position or in dynamic motion; early scapula elevation or shrugging during arm elevation; or inadequate upward and downward rotation of the scapula during arm elevation or lowering [4,5]. Scapular dyskinesis was found to be present in $61 \%$ of overhead athletes and $33 \%$ of nonoverhead athletes [6]. It has also been revealed in $67 \%$ to $100 \%$ of athletes with shoulder injuries and also in many asymptomatic athletes [6,7]. Scapular dyskinesia or altered kinematics of the scapula (downward rotation, anterior tilt, and internal rotation) subsequent to dysfunction or weakness of scapular stabilizing muscles may contribute to impingement syndrome through decreasing the subacromial space [8-10].

Subacromial impingement syndrome and rotator cuff tendinopathy account for $44 \%$ to $65 \%$ of all complaints of musculoskeletal shoulder pain, and they have been linked to scapular dyskinesis [11,12]. The researchers have suggested that scapular position and movements are altered in patients with subacromial impingement syndrome [8-10], rotator cuff tendinopathy [13], shoulder instability [14,15], and even neck pain [16]. It is not clear that the association between scapular dyskinesis and shoulder pathology represents a cause or effect of the pathology $[6,17,18]$.

The clinical evaluation of scapular motion is challenging because of the 3-dimensional scapular motion. Previous studies have described different methods of identifying scapular dyskinesis including an electromagnetic kinematic motion analysis system, 3-dimensional digitizer, visual observation, linear measurement, and manual correction maneuvers [19-22].

Exercise is a key component of shoulder rehabilitation. A systematic review by Hanratty et al [23] showed that physiotherapy exercises are effective in decreasing pain and improving function in patients with subacromial impingement syndrome at short-term follow-up. Scapular muscle exercises are included in the rehabilitation of patients with subacromial impingement syndrome and scapular dyskinesis because the muscular system is one of the major contributors of scapular positioning both at rest and during shoulder movements [24], but there is considerable uncertainty relating to the relative effectiveness of such approaches on changing scapular position and motion.

A recent systematic review and meta-analysis of 4 randomized clinical trials (RCTs) in patients with rotator cuff disorders concluded that a scapula-focused approach is more effective than generalized approaches in reducing shoulder pain up to 6 weeks, but this benefit is not apparent by 3 months. This systematic review also showed a conflicting result regarding the effect of a scapula-focused approach in comparison to generalized approaches on scapula position and movement [25]. In the aforementioned systematic review, the primary objective was to synthesize the impact of scapular intervention on rotator cuff-related shoulder pain, with the secondary objective being evaluating the effect of scapular exercises on scapula position and movement; however, it did not include gray literature, probably missing some related studies. There are some studies in which changing scapula kinematics in asymptomatic individuals has been studied.

Previous studies have shown scapular dyskinesis to be detrimental to shoulder function, and they recommend improvement or correction of abnormal scapular mechanics $[6,22,26]$. The correction of scapular dyskinesis has been recommended for decreasing the symptoms associated with shoulder pathology $[6,22,26]$, but the effects of scapular dyskinesis interventions on correcting scapular position and motion is not clear yet. Therefore, the main aim of this systematic review will be the evaluation of the effectiveness of exercise therapy on scapular position and motion in individuals with scapular dyskinesis.

\section{Objectives}

\section{Primary Objective}

- Identify the effectiveness of exercise therapy (scapular strengthening exercise, scapular stabilization exercise, scapular muscle stretching) on scapular position and motion of individuals with scapular dyskinesis

\section{Secondary Objectives}

- Identify the effectiveness of scapular exercise on shoulder pain in rotator cuff disorder patients with scapular dyskinesis

- Identify the effectiveness of scapular exercise on changing scapular kinematics in asymptomatic individuals with scapular dyskinesis

- Identify the effectiveness of scapular exercise on scapular position and motion of individuals with different types of scapular dyskinesis

- Find potential sources of heterogeneity in primary studies 


\section{Methods}

\section{Study Characteristics}

This systematic review will include any types of clinical trials (RCTs with or without concurrent control; double blind, single blind, and open-label RCTs; and before-after clinical trials) in which scapular position or motion is considered one of the main independent variables. Case studies and simulation studies will not be included.

\section{Types of Participants}

This systematic review will include studies with adult participants (aged 16 years and older, athletes and nonathletes) in which a clear diagnosis of scapular dyskinesis is defined according to any of following criteria:

\section{Abnormalities in Scapular Rest Position}

- Scapular winging: prominence of scapular medial border and/or inferior angle relative to the thoracic cage

- Scapular tilt or protraction

- SICK scapula syndrome: scapular malposition, inferior medial border prominence, coracoid pain, malposition and dyskinesis of scapular movement

\section{Abnormalities in Scapular Motion}

- Scapular dysrhythmia: early scapula elevation or shrugging during arm elevation and inadequate upward and downward rotation of the scapula during arm elevation and lowering (scapular downward rotation syndrome) [22]

The studies that focused on the effects of changing scapular kinematics in asymptomatic individuals or individuals with rotator cuff tendinopathy and subacromial impingement syndrome will be included. The studies without a clear diagnostic criterion and clinical measures for scapular dyskinesis will be excluded.

\section{Types of Intervention}

The intervention in the treatment group should be scapular focused exercises with or without general shoulder exercise. The control group will include other forms of interventions, such as manual therapy and taping, or no treatment.

Studies reporting any type of therapeutic intervention (scapular strengthening exercise, scapular stabilization exercise, scapular muscle stretching) with the aims of changing scapular biomechanics, including position and movement, and addressing the pain and disability found with scapular dyskinesis will be included. Also, scapular exercise combined with patient education and instruction on exercises will be included. Clinical trials that compare scapular kinematics after using other techniques such as manual therapy and taping and studies in which exercise has been a minor component of a multimodal approach will be excluded.

\section{Outcome Assessment}

The primary outcome will be measurements reported on scapular kinematics outcomes such as scapular rest position, static scapular positioning, scapulohumeral rhythm, and scapular dynamic control (eg, lateral scapular slide test, measurement of scapular upward and downward rotation, measurement of scapular anterior and posterior tilt, and measurement of scapular medial and lateral tilt). Our secondary outcome will include shoulder pain intensity.

\section{Information Sources}

Our electronic search database includes PubMed, Scopus, Web of Science, Elsevier, Ovid, ProQuest, Physiotherapy Evidence Database, and the Cochrane Library. The reference lists of articles, other reviews, gray literature, and key journals will be also searched for relevant articles. All studies will enter the initial screening stage without any time limit or restrictions of language and publication type.

\section{Search Strategy}

The strategy for searching will be developed and completed in the PubMed database, and then the same strategy will be applied to the other electronic databases. Textbox 1 shows the suggested PubMed search strategy.

\section{Study Records}

Two authors will search information sources independently and perform the primary article screening. At first, they will screen the titles and abstracts of all the articles independently, and then their selected articles will be categorized as eligible, not eligible, or may be eligible. When a study cannot be clearly excluded according to its title and abstract alone, its full text will be reviewed before the final decision. Articles categorized as not eligible by both reviewers will be eliminated. Each reviewer will then review the full text of the remaining articles, and a study will be included when both reviewers independently assess it as satisfying the eligibility criteria. Any disagreement during the selection of studies will be discussed and decided by the whole team.

\section{Data Extraction and Management}

Data will be extracted from papers by 2 reviewers and entered into a data extraction form. Any disagreement will be discussed and decided on by the whole team. If there are incomplete or unclear data in articles, inquiries will be sent to the authors. All searched studies will be managed through EndNote (Clarivate Analytics) software.

\section{Data Items}

From each article, the following information will be extracted: general information (author name, year of publication, journal title, date of extraction), study characteristics (title of study, study design, study setting, sample size), participant characteristics (demographics data, main inclusion criteria, sports history, healthy condition, and type of scapular dyskinesis), methods and tools of scapular dyskinesis diagnosis (observational, 2- and 3-dimensional assessment methods), intervention characteristics (exercise type, frequency, and duration), scapular outcome measurements and results (eg, lateral scapular slide test, measurement of scapular upward and downward rotation, measurement of scapular anterior and posterior tilt, and measurement of scapular medial and lateral tilt), and pain outcome measure. 
Textbox 1. Suggested PubMed search strategy.

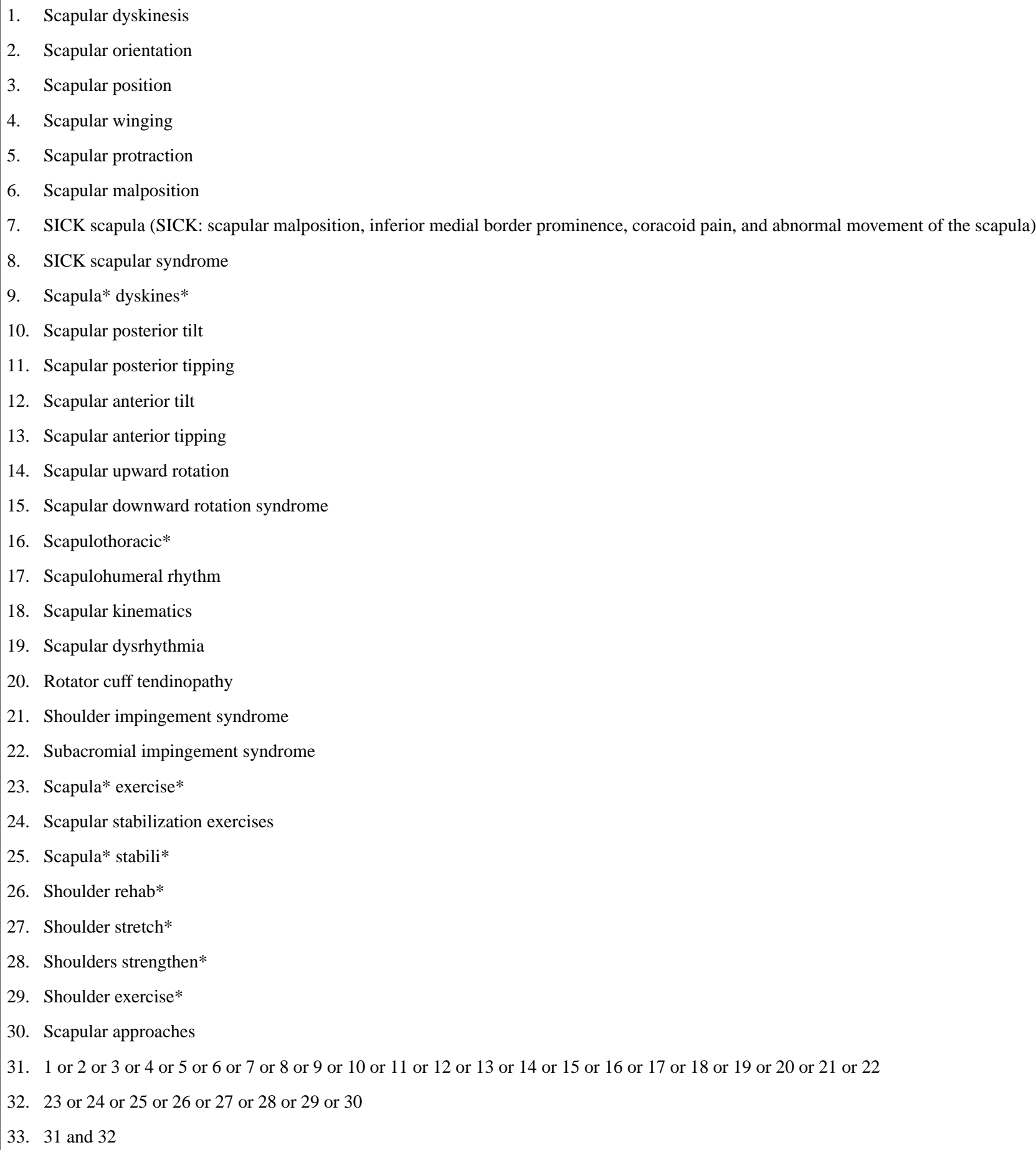

\section{Assessment of Risk of Bias in Included Studies}

The risk of bias assessment will be based on the Cochrane Collaboration Risk of Bias [27]. Risk of bias in each study will be independently assessed by 2 reviewers. Each reviewer will evaluate methodological quality using the following items: random sequence generation, allocation concealment, blinding of participants and personnel, blinding of outcome assessment, incomplete outcome data, selective reporting, and other bias. After critical appraisal of studies, each trial will be categorized into low risk, high risk, and unclear risk. In case of disagreement, discussion will take place to achieve consensus.

\section{Data Synthesis}

If enough studies are included, a meta-analysis will be conducted using the scapula and pain outcome data. For scapular outcome data, mean differences and standardized mean differences will be used for meta-analysis. In cases where data are missing, they (eg, standard deviation) will be calculated from available data (eg, standard error will be calculated from $P$ values or $95 \%$ confidence intervals) [28] or we will contact the authors.

If sufficient comparable studies are included, a subgroup analysis will be carrying out. The subgroups will be formed by quality of primary studies, type of study participants (athlete 
and nonathlete studies and asymptomatic individuals or individuals with rotator cuff-related shoulder pain), and types of scapular dyskinesis (scapular outcomes measurements and results).

\section{Results}

This systematic review began in December 2016 and is currently in progress. This is the first systematic review protocol aiming to assess the effectiveness of exercise therapy in individuals with scapular dyskinesis. The findings of this systematic review will be synthesized to determine the effectiveness of recommended therapeutic exercise on scapular position and motion in individuals with scapular dyskinesis. On completion of this project, the results of this study will be published in a peer-reviewed journal.

\section{Discussion}

Scapular dyskinesis is a condition that is commonly associated with shoulder pathology but is also present in asymptomatic individuals, and it is believed to be a risk factor for further injury [6]. Evidence suggests that patients with rotator cuff-related shoulder pain present scapular kinematic abnormalities such as decreased scapular upward rotation, decreased scapular posterior tipping, and external rotation $[8,29,30]$. It has been proposed that abnormal scapular kinematics may be linked to weakness of scapular muscles [31-33]. Specifically, increased activation of the upper trapezius with decreased activation of the lower trapezius and serratus anterior has been proposed to be related to altered scapular position and motion [34]. However, it is not clear if these differences are compensatory strategies or causative factors $[6,17,18]$.

Conservative treatment, including exercise therapy, is thought to influence various shoulder conditions and outcomes such as pain, restricted range of motion, and functional disability $[23,24]$, but there is considerable uncertainty relating to the relative effectiveness of such approaches on changing scapular position and motion. The results of this systematic review can help clinicians relating to effectiveness of therapeutic exercise on scapular dyskinesis and associated shoulder pain.

\section{Acknowledgments}

This research was supported by the Deputy of Research and Technology of the University of Social Welfare and Rehabilitation Sciences, Tehran, Iran. ANM is the guarantor of this review. ANM conceived the paper and wrote the first draft. MS, AAK, and KA contributed to development of the search study and risk of bias assessment strategy. SND and ZM contributed to the selection criteria and data abstraction criteria. All authors read and approved the final manuscript.

\section{Conflicts of Interest}

None declared.

\section{Multimedia Appendix 1}

Peer-review report.

[PDF File (Adobe PDF File), 29KB-Multimedia Appendix 1]

\section{References}

1. Lugo R, Kung P, Ma CB. Shoulder biomechanics. Eur J Radiol 2008 Oct;68(1):16-24. [doi: 10.1016/j.ejrad.2008.02.051] [Medline: 18511227$]$

2. McClure PW, Michener LA, Sennett BJ, Karduna AR. Direct 3-dimensional measurement of scapular kinematics during dynamic movements in vivo. J Shoulder Elbow Surg 2001;10(3):269-277. [doi: 10.1067/mse.2001.112954] [Medline: $\underline{11408911]}$

3. Ludewig PM, Phadke V, Braman JP, Hassett DR, Cieminski CJ, LaPrade RF. Motion of the shoulder complex during multiplanar humeral elevation. J Bone Joint Surg Am 2009 Feb;91(2):378-389 [FREE Full text] [doi: 10.2106/JBJS.G.01483] [Medline: 19181982]

4. Kibler WB, Ludewig PM, McClure PW, Michener LA, Bak K, Sciascia AD. Clinical implications of scapular dyskinesis in shoulder injury: the 2013 consensus statement from the 'Scapular Summit'. Br J Sports Med 2013 Sep;47(14):877-885. [doi: 10.1136/bjsports-2013-092425] [Medline: 23580420]

5. Kibler WB, Sciascia A. Current concepts: scapular dyskinesis. Br J Sports Med 2010 Apr;44(5):300-305. [doi: 10.1136/bjsm.2009.058834] [Medline: 19996329]

6. Burn M, McCulloch P, Lintner D, Liberman S, Harris J. Prevalence of scapular dyskinesis in overhead and nonoverhead athletes: a systematic review. Orthop J Sports Med 2016;4(2). [doi: 10.1177/2325967115627608]

7. Pluim BM. Scapular dyskinesis: practical applications. Br J Sports Med 2013 Sep;47(14):875-876. [doi: 10.1136/bjsports-2013-092722] [Medline: 23973880]

8. Ludewig PM, Cook TM. Alterations in shoulder kinematics and associated muscle activity in people with symptoms of shoulder impingement. Phys Ther 2000 Mar;80(3):276-291. [Medline: 10696154]

9. Hébert LJ, Moffet H, McFadyen BJ, Dionne CE. Scapular behavior in shoulder impingement syndrome. Arch Phys Med Rehabil 2002 Jan;83(1):60-69. [Medline: 11782834] 
10. Lopes AD, Timmons MK, Grover M, Ciconelli RM, Michener LA. Visual scapular dyskinesis: kinematics and muscle activity alterations in patients with subacromial impingement syndrome. Arch Phys Med Rehabil 2015 Feb;96(2):298-306. [doi: 10.1016/j.apmr.2014.09.029] [Medline: 25449194]

11. van der Windt DA, Koes BW, de Jong BA, Bouter LM. Shoulder disorders in general practice: incidence, patient characteristics, and management. Ann Rheum Dis 1995 Dec;54(12):959-964 [FREE Full text] [Medline: 8546527$]$

12. Vecchio P, Kavanagh R, Hazleman BL, King RH. Shoulder pain in a community-based rheumatology clinic. Br J Rheumatol 1995 May;34(5):440-442. [Medline: 7788173]

13. Ludewig PM, Reynolds JF. The association of scapular kinematics and glenohumeral joint pathologies. J Orthop Sports Phys Ther 2009 Feb;39(2):90-104 [FREE Full text] [doi: 10.2519/jospt.2009.2808] [Medline: 19194022]

14. von Eisenhart-Rothe R, Matsen FA, Eckstein F, Vogl T, Graichen H. Pathomechanics in atraumatic shoulder instability: scapular positioning correlates with humeral head centering. Clin Orthop Relat Res 2005 Apr(433):82-89. [Medline: 15805941]

15. Illyés A, Kiss RM. Kinematic and muscle activity characteristics of multidirectional shoulder joint instability during elevation. Knee Surg Sports Traumatol Arthrosc 2006 Jul;14(7):673-685. [doi: 10.1007/s00167-005-0012-7] [Medline: 16362361]

16. Helgadottir H, Kristjansson E, Mottram S, Karduna AR, Jonsson H. Altered scapular orientation during arm elevation in patients with insidious onset neck pain and whiplash-associated disorder. J Orthop Sports Phys Ther 2010 Dec;40(12):784-791. [doi: 10.2519/jospt.2010.3405] [Medline: 20972341]

17. Kibler WB. The role of the scapula in athletic shoulder function. Am J Sports Med 1998;26(2):325-337. [doi: 10.1177/03635465980260022801] [Medline: 9548131]

18. Myers J, Oyama S, Hibberd E. Scapular dysfunction in high school baseball players sustaining throwing-related upper extremity injury: a prospective study. J Shoulder Elbow Surg 2013;22(9):1154-1159. [doi: 10.1016/j.jse.2012.12.029]

19. Kibler W, Uhl T, Maddux J, Brooks P, Zeller B, McMullen J. Qualitative clinical evaluation of scapular dysfunction: a reliability study. J Shoulder Elbow Surg 2002;11(6):550-556. [doi: 10.1067/mse.2002.126766]

20. Uhl TL, Kibler WB, Gecewich B, Tripp BL. Evaluation of clinical assessment methods for scapular dyskinesis. Arthroscopy 2009 Nov;25(11):1240-1248. [doi: 10.1016/j.arthro.2009.06.007] [Medline: 19896045]

21. McClure P, Tate AR, Kareha S, Irwin D, Zlupko E. A clinical method for identifying scapular dyskinesis, part 1: reliability. J Athl Train 2009;44(2):160-164 [FREE Full text] [doi: 10.4085/1062-6050-44.2.160] [Medline: 19295960]

22. Struyf F, Nijs J, Mottram S, Roussel N, Cools A, Meeusen R. Clinical assessment of the scapula: a review of the literature. Br J Sports Med 2014;48(11):883-890. [doi: 10.1136/bjsports-2012-091059]

23. Hanratty C, McVeigh J, Kerr D, Basford J, Finch M, Pendleton A. The effectiveness of physiotherapy exercises in subacromial impingement syndrome: a systematic review and meta-analysis. Semin Arthritis Rheum 2012;42(3):297-316. [doi: 10.1016/j.semarthrit.2012.03.015]

24. Zhang M, Zhou J, Zhang Y, Wang J, Zhang Q, Chen W. Clinical effectiveness of scapulothoracic joint control training exercises on shoulder joint dysfunction. Cell Biochem Biophys 2015 May;72(1):83-87. [doi: 10.1007/s12013-014-0408-4] [Medline: 25416584]

25. Bury J, West M, Chamorro-Moriana G, Littlewood C. Effectiveness of scapula-focused approaches in patients with rotator cuff related shoulder pain: a systematic review and meta-analysis. Man Ther 2016;25:35-42. [doi: 10.1016/j.math.2016.05.337]

26. Harris J, Pedroza A, Jones G. Predictors of pain and function in patients with symptomatic, atraumatic full-thickness rotator cuff tears: a time-zero analysis of a prospective patient cohort enrolled in a structured physical therapy program. Am J Sports Med 2012;40(2):359-366. [doi: 10.1177/0363546511426003]

27. Higgins J, Altman D, Gotzsche P, Juni P, Moher D, Oxman A. The Cochrane Collaboration's tool for assessing risk of bias in randomised trials. BMJ 2011;343. [doi: 10.1136/bmj.d5928]

28. Higgins J, Green S. Cochrane Handbook for Systematic Reviews of Interventions 4.2.5.: The Cochrane Library; 2005. URL: http://training.cochrane.org/sites/training.cochrane.org/files/public/uploads/resources/Handbook5 1/Handbook4.2. 6Sep2006.pdf [accessed 2017-11-15] [WebCite Cache ID 6uzsZCK2C]

29. McClure PW, Michener LA, Karduna AR. Shoulder function and 3-dimensional scapular kinematics in people with and without shoulder impingement syndrome. Phys Ther 2006 Aug;86(8):1075-1090. [Medline: 16879042]

30. Timmons M, Thigpen C, Seitz A, Karduna A, Arnold B, Michener L. Scapular kinematics and subacromial-impingement syndrome: a meta-analysis. J Sport Rehabil 2012 Nov;21(4):354-370. [Medline: 22388171]

31. Cools AM, Witvrouw EE, Declercq GA, Vanderstraeten GG, Cambier DC. Evaluation of isokinetic force production and associated muscle activity in the scapular rotators during a protraction-retraction movement in overhead athletes with impingement symptoms. Br J Sports Med 2004 Feb;38(1):64-68 [FREE Full text] [Medline: 14751949]

32. Cools AM, Witvrouw EE, Mahieu NN, Danneels LA. Isokinetic scapular muscle performance in overhead athletes with and without impingement symptoms. J Athl Train 2005 Jun;40(2):104-110 [FREE Full text] [Medline: 15970956]

33. Leong H, Tsui S, Ng G, Fu S. Reduction of the subacromial space in athletes with and without rotator cuff tendinopathy and its association with the strength of scapular muscles. J Sci Med Sport 2016 Dec;19(12):970-974. [doi:

10.1016/j.jsams.2016.03.011] [Medline: 27102401] 
34. Huang T, Ou H, Huang C, Lin J. Specific kinematics and associated muscle activation in individuals with scapular dyskinesis. J Shoulder Elbow Surg 2015 Aug;24(8):1227-1234. [doi: 10.1016/j.jse.2014.12.022] [Medline: 25704212]

\section{Abbreviations}

RCT: randomized clinical trial

SICK: scapular malposition, inferior medial border prominence, coracoid pain, and abnormal movement of the scapula

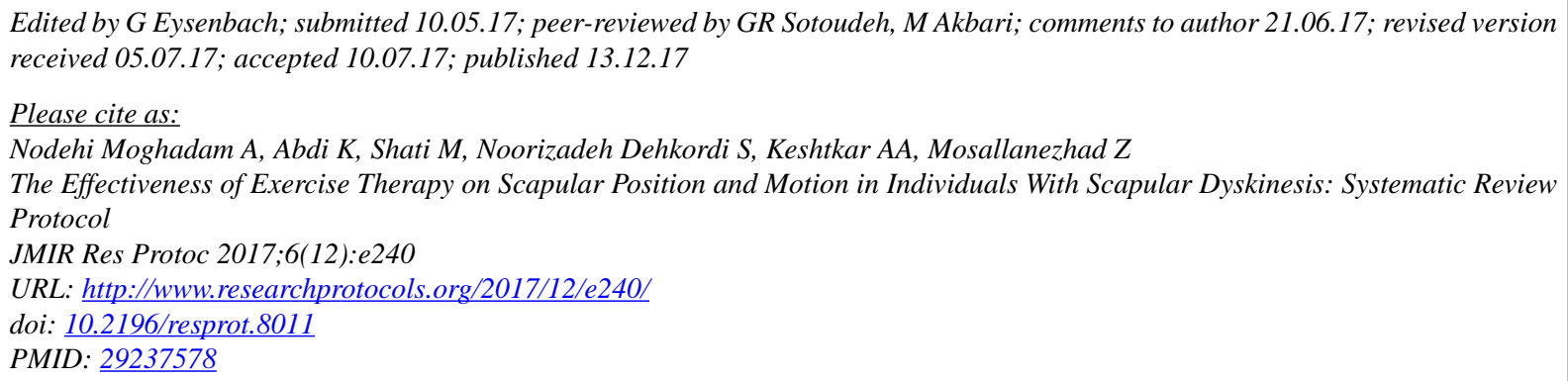

(C)Afsun Nodehi Moghadam, Kianoush Abdi, Mohsen Shati, Shohreh Noorizadeh Dehkordi, Abbas Ali Keshtkar, Zahra Mosallanezhad. Originally published in JMIR Research Protocols (http://www.researchprotocols.org), 13.12.2017. This is an open-access article distributed under the terms of the Creative Commons Attribution License (https://creativecommons.org/licenses/by/4.0/), which permits unrestricted use, distribution, and reproduction in any medium, provided the original work, first published in JMIR Research Protocols, is properly cited. The complete bibliographic information, a link to the original publication on http://www.researchprotocols.org, as well as this copyright and license information must be included. 